\title{
Orthorexia nervosa and dieting in a non-clinical sample: a prospective study
}

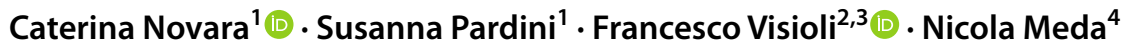

Received: 17 October 2021 / Accepted: 21 December 2021 / Published online: 7 January 2022

(c) The Author(s) 2022

\begin{abstract}
Purpose Orthorexia Nervosa $(\mathrm{ON})$ is characterised by excessive attention to a dietary regimen perceived as healthy. A critical factor in the distinction between $\mathrm{ON}$ and other eating disorders (EDs) is the dichotomy of quality-versus-quantity of food intake. We investigated whether specific types of diet or dieting frequency are associated with orthorexic features, explored the overlap between ON and EDs symptoms, and examined which constructs are predictive of ON after 6 months. Methods A total of 1075 students (75.1\% female, mean age 20.9) completed a set of questionnaires assessing Orthorexia, Eating Disorders, Obsessions and Compulsions, Anxiety and Depression; 358 individuals (79.9 female, mean age 20.9) agreed to participate in the study and completed the same questionnaires after 6 months. Different regression models were defined to investigate our hypothesis.

Results Findings suggest that $\mathrm{ON}$ is associated with the number and type of diets followed over a lifetime. Moreover, participants with EDs, body dissatisfaction, or a dysfunctional idea of thinness are more likely to report a greater degree of ON features. After 6 months, the best predictors of ON characteristics are the same ON characteristics assessed at the first administration, with a significant role in the ideal of thinness.

Conclusions $\mathrm{ON}$ is more frequent in individuals with a previous diagnosis of EDs and in individuals who followed a restrictive diet or a vegan/vegetarian one; the number of lifetime diets, beliefs, and behaviors related to the ideals of thinness or body dissatisfaction is common features of ON. Moreover, considering that having ON features in the past is the best ON predictor in the present, we can presume that $\mathrm{ON}$ is a construct stable over time.

Level of evidence Level IV: Evidence obtained from multiple time series analysis such as case studies. (NB: Dramatic results in uncontrolled trials might also be regarded as this type of evidence).
\end{abstract}

Keywords Mental health $\cdot$ Young adults $\cdot$ Prospective study $\cdot$ Eating behaviour $\cdot$ Dietary pattern $\cdot$ Orthorexia nervosa

\section{Introduction}

Orthorexia Nervosa $(\mathrm{ON})$ is an unhealthy eating behavior characterised by an excessive concern for a healthy diet or food quality [1]. Although not officially considered a mental

Caterina Novara

caterina.novara@unipd.it

1 Department of General Psychology, University of Padova, Via Venezia 8, 35131 Padova, Italy

2 Department of Molecular Medicine, University of Padova, Viale G. Colombo 3, Padova, Italy

3 IMDEA-Food, CEI UAM + CSIC, Carr. de Canto Blanco 8, E, Madrid, Spain

4 Department of Medicine, University of Padova, Via Giustiniani 2, Padova, Italy disorder, and its conceptualisation is controversial [2-5], some standard criteria for diagnosis of $\mathrm{ON}$ have been proposed and can be summarised as follows: (i) recurrent worry about healthy dieting or food quality; (ii) excessive focus on strict dietary rules and emotional consequences when transgressed [2]; and (iii) significant impairment of daily life activities and distress due to $\mathrm{ON}[5,6]$. ON prevalence rate varied widely in previous studies (from $1 \%$ to over $88 \%$ ) due to the controversial definition or heterogenous assessment instruments $[7,8]$.

A central feature of the current debate on $\mathrm{ON}$ concerns the differentiation between a pathological condition and a healthy lifestyle that entails an interest in proper food choices and weight control through healthy behaviors. Excessive attention to a healthy lifestyle might have medical and psychological consequences [6], yet many such 
behaviors are widely promoted by mass media and often endorsed by Western societies $[9,10]$. In addition, the overlap with some diagnoses recognised as Eating Disorders (EDs) (e.g., Anorexia Nervosa (AN) or Bulimia Nervosa (BN)) and Obsessive-Compulsive Disorder (OCD) [11] make ON a multi-faceted and evolving framework.

Much research has highlighted a significant intersection between ON and EDs [12]. Otherwise, the distinctions between EDs and ON imply the dichotomy of quantity-versus-quality: while individuals with EDs are more concerned about food intake, those with ON are worried about food quality [6]. Furthermore, weight reduction for health reasons has been underscored in the ON framework [13]. Some qualitative clinical studies on hospitalised individuals have highlighted how the $\mathrm{ON}$ is associated with a diet for weight loss or dictated by health problems [2, 14, 15]. In addition, a study on individuals who have AN and BN features showed a high prevalence of $\mathrm{ON}$ characteristics that tend to increase after treatment [16]. Regarding the non-clinical population, particular attention has been paid to groups considered at risk for ON (physicians, dieticians, medical/nutrition/dietetic's students, and individuals who followed diets for moral/ healthy reasons [13, 17-22]). It is also important to consider previous and current dietary habits, the type and reasons for dieting, and the overlap with past EDs to investigate similarities and differences between ON and EDs in the non-clinical population.

Most of the literature regarding ON administered the ORTO-15 questionnaire [23]. Even if a cutoff has been extrapolated, the questionnaire does not differentiate healthy from problematic eating patterns [24-26]. Because of this, reports on the relationships between age, gender, Body Mass Index (BMI), and ON characteristics have been inconsistent. Some studies have shown that $\mathrm{ON}$ is more common among young individuals [27-29], whereas others [23, 30] have reported that orthorexic characteristics are more likely to emerge along with age. Yet, other investigations did not highlight any significant relationship between age and ON [31-33]. Cross-national studies reported that $\mathrm{ON}$ is more common in women [28, 34-37], although the female gender is often predominant in cohorts [38]. Three studies have shown that $\mathrm{ON}$ is more frequent in men [23, 27, 39], while other authors reported no relationship between gender and ON [26, 33, 40-44]. Data on BMI are also inconsistent [38]: while some research highlighted no associations between BMI and ON [1, 23, 33, 35, 37, 39, 45-47], several authors reported that a high BMI is related to the risk of ON [1, $27,31,34,36,42,48,49]$, or that a low BMI is a predictor of ON features [47, 50, 51]. A recent review highlighted that the relationship between ON and BMI is not clear [38]. Thus, it might be essential to consider the role of BMI in ON with valid instruments and within more multifaceted framework related to diet, the presence of physical and psychological disorders, or constructs associated with EDs. The Eating Habits Questionnaire (EHQ) [52] has noteworthy psychometric properties. In particular, it is composed of three scales: Knowledge, Feelings, and Problems. Although it does not yet have a cutoff threshold, the Problems subscale seems to differentiate disordered eating from healthy eating, as it refers to the specific interferences and problems due to the adhesion to diet. In addition, the Problems subscale has shown a convergent validity with EDs symptoms, obsessive-compulsive symptoms, and depression [15, 24, 52].

Most of the literature published thus far has focused on samples of individuals that follow a specific diet and investigated the quality of the diets pursued and the motivations that led to this choice (moral in nature, health, or weight loss [22, 53]). These populations might have constantly been dieting or had undertaken numerous diets in their lives that could have exacerbated ON behavior, hence broadly explaining the incidence of $\mathrm{ON}$.

This work aimed at assessing whether the frequency and type of diet are associated with orthorexic features, ascertain the ON and EDs overlap, and explore which of these constructs are predictive of $\mathrm{ON}$ characteristics after a time frame of 6 months. We addressed these issues from a perspective viewpoint to understand which aspects related to $\mathrm{ON}$ are pivotal in predicting the symptomatology of $\mathrm{ON}$ after some time. In line with Zikgraf et al., [24], we anticipated that the EDs-related symptomatology is more associated with functional impairments. We also expect the number and qualities of diets (i.e., vegetarians, vegans) to be associated with any ON construct $[32,54,55]$.

\section{Methods}

\section{Study cohort}

All procedures were approved by the University of Padova Psychology Ethical Committee (Area 17) under the latest version of the Declaration of Helsinki, and participants provided written informed consent. Recruitment took place in Padova, a city in North-Eastern Italy, between October 2019 and June 2020. At the start of different teaching classes, we presented, either in person or via pre-recorded videos, the aims of the prospective study [56] to approximately 8,000 University of Padova's students. The students were handed out a card containing an URL. By accessing the URL, students could provide their informed consent and participate in the study. Every 6 months, the participants were automatically asked by email to participate in another data collection. A total of 1,477 students agreed to participate in the study (18.4\% response rate). Of this pool, 1,075 participants matched the target population characteristics (Italian native speaker students aged 18-30); participants were excluded 
if non-native speakers outside the previous age range or partially completed the questionnaires. No other inclusion or exclusion criteria were applied, and completed a demographic schedule and questionnaires based on the RedCap web application [57].

\section{Measures}

Demographics schedule: the participants were asked to report their gender, age, BMI (calculated by self-reported height and weight), years of education, marital status, and occupation. Moreover, participants had to report if they had ever been diagnosed with a mental health condition or other medical disorder/illness/disease or if they had a family history of mental health issues. Participants were asked to report (a) how many diets they had followed in their lifetime; (b) prescribed diets, self-created, or inspired by a diet found on the internet or in books; (c) time since the last diet, and what type of diet (prescribed, self-created); (d) if they had any food intolerance and-if so-to what type of food; e) if they avoided a specific type of food and why; and d) if they had some physical illness (such as metabolic or gastroenterological issues).

The Beck Depression Inventory-II (BDI-II) is a 21-item self-report scale that assesses the severity of affective, cognitive, motivational, vegetative, and psychomotor features of depression; it has been thoroughly validated [58-61]. It is based on a four-point Likert scale, scored from 0 (absent) to 3 (severe), and total scores range from 0 to 63 . The Italian version showed good internal consistency, considering a sample of university students, patients with depression, and a group of individuals extracted from the general population $(0.80<$ Cronbach's $\alpha<0.87)$. The test-retest reliability across a period of a month was good $(r=0.76)$, as well as good convergent, divergent, and criterion validity [60]. Our sample maintained a high internal consistency (BDI-II $\alpha=0.91$ ).

The Beck Anxiety Inventory (BAI) [62-65] is a 21-item self-report that measures physiological and cognitive anxiety symptoms on a four-point Likert scale scored from 0 (not at all) to 3 (severely), and total scores range from 0 to 63 . The Italian version showed good psychometric properties. In a sample of students, its internal consistency (Cronbach's $\alpha=0.89$ ) and the test-retest reliability were good as well as for construct validity [65]. In the present study, high internal consistency has been shown $(\alpha=0.92)$.

The Obsessive-Compulsive Inventory-Revised (OCI-R) $[66,67]$ is an 18 -item self-report questionnaire assessing the Obsessive-Compulsive Disorder (OCD) symptoms on a five-point Likert scale (Not at all, A little, Moderately, A lot and Extremely), and total scores range from 0 to 72 . The questionnaire comprises six subscales (Washing, Ordering, Hoarding, Mental Neutralizing, Obsessing, and Checking) composing an additional final total score. The original version has good reliability and validity indices of the OCI$\mathrm{R}$, showing strong convergence with established measures of OCD, moderate to high internal consistency across the six subscales, and adequate to high test-retest stability. Regarding the Italian version [67], the confirmatory factor analysis showed the original six-factor structure. Moreover, a good internal consistency is confirmed $(0.76<$ Cronbach's $\alpha<0.94)$. A 30-day test-retest reliability was good $(0.76<\mathrm{r}<0.99)$ and convergent, discriminant and criterion validity were acceptable [67]. In this study, the questionnaire's acceptable internal consistency is highlighted both for the total $(\alpha=0.88)$ and the subscales scores (Cronbach's $\alpha$ ranging from 0.67 - "Mental Neutralizing" and "Checking" subscales-to 0.86 "Obsessing").

The Eating Disorder Inventory - 3 (EDI-3) [68-70] is a 91-item self-report questionnaire investigating symptoms of Eating Disorders and other related psychological features on a six-point Likert scale from 1 (Never) and 6 (Always). The EDI-3 is composed of twelve subscales. Some of them are related to EDs' symptoms (i.e., Drive for Thinness, Bulimia, and Body Dissatisfaction) and are grouped in the "Eating Disorder Risk". The other scales are about psychological features related to EDs (Low Self-Esteem, Personal Alienation, Interpersonal Insecurity, Interpersonal Alienation, Interoceptive Deficits, Emotional Dysregulation, Perfectionism, Asceticism, and Maturity Fears), and are grouped in the "General Maladjustment" scale. The present study categorised the participants in different manifestations of symptoms' severity (as described in [68]) of eating disorders. For the General Maladjustment subscale, we considered that a score below 37 (25th percentile) means mild or absent symptomatology, whereas a score above 80 (66th percentile) marks the presence of severe symptoms. Between these two cutoffs, the score underlies moderate symptomatology. For the Eating Disorder Risk subscale, a score below 12 (25th percentile) is associated with mild or absent symptomatology, whereas a score above 36 (66th percentile) indicates the presence of severe symptoms. A score between 12 and 36 is associated with moderate symptomatology.

Good internal consistency was highlighted in the Italian version $(0.72<$ Cronbach's $\alpha<0.94)$ [69], as well as a good day test-retest reliability, cross-informant agreement, and a good discriminating validity $[68,69]$. In the current study, Cronbach $\alpha$ was high for the total score and its subscales, i.e., total $\alpha=0.96$, General Maladjustment $\alpha=0.95$, and ED Risk $\alpha=0.94$ ).

The Eating Habits Questionnaire (EHQ) [52-71] is a 21-item self-report questionnaire aimed to assess ON characteristics on a four-point Likert scale from 1 to 4 ("false, not at all true," "slightly true," "mainly true," and "very true"); total scores range from 0 to 84 . It is characterised by three subscales (Knowledge, Feelings, and Problems), 
which refer to the negative impact that the participants' diet style has had on the family, work, social and daily life (i.e., "I go out less since I began eating healthily"). The Italian validation, exploratory, and confirmatory factorial analysis evidence the same original EHQ structure [71]. Moreover, a good internal consistency and a 1-month test-retest reliability were highlighted ( $\mathrm{r}$ ranging from 0.50 to $0.75 ; 0.001<\mathrm{p}<0.01)$. The original and Italian versions highlighted adequate internal consistency indices and convergent and divergent validity [52, 71]. Based on the preliminary analysis conducted on Italian samples, including both groups of individuals with clinical problems and extracted from the general population, a score higher than 50 on the EHQ and corresponding to the 90th percentile could be considered helpful in discriminating individuals with high and low orthorexic features $[15,71]$. In this study, the reliability was acceptable (for total score $\alpha=0.87$; Problems subscale $\alpha=0.85$; Knowledge subscale $\alpha=0.76$; Feelings subscale $\alpha=0.64$ after removing item 9 - see also the Appendix).

\section{Statistical analysis}

Anonymised data were downloaded from the REDCap [72] platform and curated using RStudio 3.5.3 [73-76]. Participants were divided into different severity classes according to their scores on the EDI-3 questionnaire [68, 69]; this is a practical approach for distinguishing the role of symptom severity on the studied outcome $[68,69]$.

In this work, we investigated the variables associated with characteristics of orthorexia with the EHQ. We analysed the factors associated with higher scores (i.e., more severe characteristics) as described below. To assess the association of the EHQ total and subscales scores with demographic factors and variables related to personal life, such as the history of mental disorders, eating behaviors, we defined different regression models (generalised linear mixed models, GLMMs). We used the Poisson family of distributions to model the data (as employed in [76-79] for count data with left-skewed distributions). Here we report only the models with the lowest Akaike Information Criterion (AICan index of model fitting: the lower the AIC, the lower the variance left unexplained by the model, the better the fit) identified with a stepwise selection approach. We report the $\beta$ estimate for each variable of the model with the lowest AIC. The estimation of a variable represents the importance of that variable in changing the questionnaire scores. We ensured that scores were not better predictors of orthorexia symptomatology before categorising - symptom severity into classes. To assess what factors were associated with a relevant score (i.e., $>=50$ ) of the EHQ questionnaire, we defined a binomial regression model and applied the same principles of analysis described above for the other GLMMs.

Sample characteristics and regression models employed are reported in Tables 1 and 2 and in the Appendix, respectively.

\section{Results}

Of the 1,075 participants who completed the questionnaires at t-zero, 358 completed the questionnaires 6 months later. Students that participated in the study at t-zero were enrolled in Medicine and Surgery (37.8\%), Science, Technology, Engineering, or Mathematics (21.9\%), Biology, Pharmacy, or other Life Sciences (12.7\%), Social and Political Sciences, or Law or Economics (10.7\%), Psychology (9\%), Arts and Humanities (7.9\%). Students who were married or cohabiting were $2.7 \%(n=29)$, while $97.3 \%$ were single or in a relationship. Only $3.2 \%$ of the participants worked (fulltime or part-time or project work), whereas $96.8 \%$ were fulltime students. As reported elsewhere [56], the lockdowns that followed the coronavirus pandemic did not significantly influence the EHQ scores in these participants.

The group of students who participated in the follow-up did not significantly differ in the prevalence of self-reported $\mathrm{AN}$ and $\mathrm{BN}$ from participants who did not participate in the follow-up. Moreover, we tested the prevalence of the disorders in the two groups at the first cross-sectional, knowing which participants would eventually participate or not in the second cross-sectional; AN: Pearson's $\chi^{2}(1, N=1075)=3.009, p=0.08$; BN: Pearson's $\chi^{2}(1$, $N=1075)=0.25, p=0.61)$. However, the prevalence of history of major depressive disorder was higher in the group that took part in the second cross-sectional (Pearson's $\chi^{2}$ $(1, N=1075)=7.007, p=0.008)$. Females percentage of participants was higher in the sample that completed the second wave than in the sample that participated only in the first wave (Pearson's $\chi 2(1, N=1075)=6.42, p=0.01)$. The comparisons for other relevant characteristics are not statistically significant and are reported in the Appendix.

\section{ON, diets and ED characteristics}

For each subscale and the EHQ total score, we defined several regression models. We tested the role of each variable reported by the participants to determine what individual characteristics significantly altered the scores of the questionnaires, i.e., were associated with more severe characteristics.

Several factors were associated with higher scores at the Problems subscale (Fig. 1A and Appendix Table 1): commitment to a vegetarian/vegan diet was associated with more social, work or individual problems than being 
Table 1 Sample characteristics

\begin{tabular}{|c|c|c|c|c|c|}
\hline & $\begin{array}{l}\text { Females w/out past } \\
\text { ED }\end{array}$ & Females with past ED & Females (total) & Males & All participants \\
\hline No. participants (\%) & $754(93,3 \%)$ & $54(6,7 \%)$ & $808(75,1 \%)$ & $267(24,9 \%)$ & 1075 \\
\hline Age $($ mean \pm sd $)$ & $20.9 \pm 2.03$ & $21.1 \pm 2.21$ & $20.9 \pm 2.04$ & $21 \pm 2.23$ & $20.9 \pm 2.09$ \\
\hline $\begin{array}{l}\text { Normal weight [No. } \\
(\%)]\end{array}$ & $647(85,9 \%)$ & $39(72,3 \%)$ & $686(85 \%)$ & $214(80,4 \%)$ & $900(83,8 \%)$ \\
\hline $\begin{array}{l}\text { Underweight [No. } \\
(\%)]\end{array}$ & $55(7,3 \%)$ & $8(14,8 \%)$ & $63(7,8 \%)$ & $9(3,3 \%)$ & $72(6,6 \%)$ \\
\hline Overweight [No. (\%)] & $42(5,5 \%)$ & $6(11,1 \%)$ & $48(5,9 \%)$ & $40(14,9 \%)$ & $88(8,2 \%)$ \\
\hline Obese [No. (\%)] & $10(1,3 \%)$ & $1(1,8 \%)$ & $11(1,3 \%)$ & $4(1,4 \%)$ & $15(1,4 \%)$ \\
\hline $\begin{array}{l}\text { EHQ Total (mean } \pm \\
\text { sd; median, [IQR]) }\end{array}$ & $\begin{array}{l}40.8 \pm 8.75 ; 40 \\
\quad[35-46]\end{array}$ & $\begin{array}{l}50.8 \pm 9.66 ; 51,[45- \\
58.8]\end{array}$ & $\begin{array}{l}41.5 \pm 9.15 ; 40 \\
\quad[35-47]\end{array}$ & $\begin{array}{l}39.5 \pm 7.97 ; 38 \\
\quad[34-44]\end{array}$ & $41 \pm 8.91 ; 40,[35-46]$ \\
\hline EHQ knowledge & $\begin{array}{l}12.8 \pm 3.08 ; 13 \\
{[11-15]}\end{array}$ & $\begin{array}{l}14.0 \pm 3.07 ; 15 \\
{[13-16]}\end{array}$ & $\begin{array}{l}12.9 \pm 3.09 ; 13 \\
{[11-15]}\end{array}$ & $\begin{array}{l}12.6 \pm 2.94 ; 13 \\
{[10-15]}\end{array}$ & $\begin{array}{l}12.8 \pm 3.06 ; 13,[11- \\
15]\end{array}$ \\
\hline EHQ feelings & $\begin{array}{l}10.2 \pm 2.59 ; 10 \\
{[9-12]}\end{array}$ & $\begin{array}{l}11.4 \pm 2.07 ; 11,[10- \\
12.8]\end{array}$ & $\begin{array}{l}10.3 \pm 2.57 ; 10 \\
{[9-12]}\end{array}$ & $\begin{array}{l}9.79 \pm 2.32 ; 10 \\
\quad[8-11]\end{array}$ & $10.1 \pm 2.52 ; 10$ \\
\hline EHQ problems & $\begin{array}{l}17.8 \pm 5.2 ; 16,[14- \\
20]\end{array}$ & $\begin{array}{l}25.4 \pm 7.49 ; 25,[19- \\
\quad 30.8]\end{array}$ & $\begin{array}{l}18.3 \pm 5.7 ; 17,[14- \\
21]\end{array}$ & $\begin{array}{l}17.2 \pm 4.55 ; 16 \\
{[14-19]}\end{array}$ & $\begin{array}{l}18.0 \pm 5.46 ; 16,[14- \\
20]\end{array}$ \\
\hline Drive for thinness & $8.45 \pm 7.25 ; 6,[2-13]$ & $\begin{array}{l}15.4 \pm 7.50 ; 16 \\
{[10-21]}\end{array}$ & $8.91 \pm 7.47 ; 7,[3-14]$ & $4.36 \pm 4.74 ; 3,[1-5]$ & $7.78 \pm 7.16 ; 5,[2-12]$ \\
\hline Vegans [No. (\%)] & $7(0,9 \%)$ & $2(3,7 \%)$ & $9(1,1 \%)$ & 0 & $9(0,8 \%)$ \\
\hline Vegetarians [No. (\%)] & $39(5,1 \%)$ & $5(9,2 \%)$ & $44(5,4 \%)$ & $5(1,8 \%)$ & $49(4,5 \%)$ \\
\hline $\begin{array}{l}\text { With anorexia nervosa } \\
\text { [No. }(\%)]\end{array}$ & $/ /$ & // & $39(4,8 \%)$ & 0 & $39(3,6 \%)$ \\
\hline $\begin{array}{l}\text { With bulimia nervosa } \\
{[\text { No. }(\%)]}\end{array}$ & $/ /$ & // & $18(2,2 \%)$ & 0 & $18(1,6 \%)$ \\
\hline $\begin{array}{l}\text { With a physical ill- } \\
\text { ness/disease/disor- } \\
\text { der [No. }(\%)]\end{array}$ & $121(16 \%)$ & $11(20,3 \%)$ & $132(16,3 \%)$ & $29(10,8 \%)$ & $161(14,9 \%)$ \\
\hline $\begin{array}{l}\text { Followed at least one } \\
\text { diet }[\text { No. }(\%)]\end{array}$ & $374(49,6 \%)$ & $51(94,4 \%)$ & $425(52,5 \%)$ & $96(35,9 \%)$ & $521(48,4 \%)$ \\
\hline
\end{tabular}

Participants with a BMI lower than 18 have underweight. Participants with a BMI higher than 25.0 but lower than 30.0 are considered to have overweight. Participants with a BMI equal to or higher than 30 have at least Grade I Obesity

$E D$ eating disorder(s), $E H Q$ eating habits questionnaire, $I Q R$ interquartile range

"omnivore" (vegetarian: $\beta=0.13$, CI 95\% (0.07-0.2), $\mathrm{p}<0.001$; vegan: $\beta=0.16$, CI 95\% (0.03-0.3), $p<0.05)$. Participants who had followed (or were following at the time of testing) at least one diet in their lifetime had more severe ON characteristics than those who had never followed any dietary regimen $(\beta=0.15$, CI 95\% (0.12-0.19), $p<0.001)$. Participants who self-reported a diagnosis of any physical illness, disease, or disorder were also more likely to have higher scores $(\beta=0.06$, CI 95\% (0.02-0.1), $p<0.01)$, as well as participants with a history of bulimia nervosa $(\beta=0.15$, CI 95\% $(0.05-0.25), p<0.001)$ or anorexia nervosa $(\beta=0.2$, CI 95\% $(0.13-0.27), p<0.001)$. We also evidenced a bias in the score against male participants, who suffered more severe $\mathrm{ON}$ characteristics than females $(\beta=0.08$, CI 95\% (0.04-0.12), $p<0.001)$, even though none of the male participants reported suffering or having suffered from an eating disorder. Among the other questionnaires, the EDI-III subscales (ED Risk and
General Maladjustment) scores were significantly associated with higher scores at the EHQ Problems subscale (Maladjustment, moderate symptoms: $\beta=0.04$, CI $95 \%$ (0.0-0.09), $p<0.05$; severe symptoms: $\beta=0.08$, CI $95 \%$ (0.04-0.13), $p<0.001$. ED Risk, moderate symptoms: $\beta=0.06$, CI 95\% (0.02-0.1), $p<0.01$; severe symptoms: $\beta=0.21$, CI $95 \%(0.15-0.26), p<0.001)$. The only factor associated with less severe symptomatology was BMI: for every 10 points increase of BMI, the score was reduced by $\beta=-0.17$ (CI 95\% -0.23 to $-0.12, p<0.001$ ).

Feelings related to food and diet were more pronounced in participants who had more severe eating disorder symptoms, as measured by the scores on the ED Risk subscale (Fig. 1B and Appendix Table 2A. See Appendix Table 2B for model characteristics when also considering item 9 in the Feelings score. Moderate symptoms: $\beta=0.07$, CI $95 \%$ (0.02-0.13), $p<0.01$; severe symptoms: $\beta=0.15$, CI $95 \%$ $(0.08-0.21), p<0.001)$, or in participants who reported 
Table 2 Characteristics, at t-zero, of the students that also participated in the 2 nd administration

\begin{tabular}{|c|c|c|c|c|c|}
\hline & $\begin{array}{l}\text { Females w/out past } \\
\text { ED }\end{array}$ & Females with past ED & Females (total) & Males & All participants \\
\hline $\begin{array}{l}\text { No. participants (\%) } \\
\text { to } 2 \text { nd cross-sec- } \\
\text { tional; characteris- } \\
\text { tics at } 1 \text { st cross- } \\
\text { sectional }\end{array}$ & $262(91,6 \%)$ & $24(8,4 \%)$ & $286(79,9 \%)$ & $72(20,1 \%)$ & $\begin{array}{l}358\left(33,3 \% \text { of } 1^{\text {st }} \text { cross- }\right. \\
\text { sectional participants })\end{array}$ \\
\hline Age $($ mean $\pm s d)$ & $20.8 \pm 2.09$ & $21.2 \pm 2.63$ & $20.8 \pm 2.14$ & $21.1 \pm 2.16$ & $20.9 \pm 2.14$ \\
\hline $\begin{array}{l}\text { Normal weight [No. } \\
(\%)]\end{array}$ & $219(83,8 \%)$ & $17(70,9 \%)$ & $236(82,6 \%)$ & $63(87,6 \%)$ & $299(83,7 \%)$ \\
\hline $\begin{array}{l}\text { Underweight [No. } \\
(\%)]\end{array}$ & $24(9,1 \%)$ & $5(20,8 \%)$ & $29(10,1 \%)$ & $2(2,7 \%)$ & $31(8,6 \%)$ \\
\hline Overweight [No. (\%)] & $18(6,8 \%)$ & $2(8,3 \%)$ & $20(7 \%)$ & $6(8,3 \%)$ & $26(7,2 \%)$ \\
\hline Obese [No. (\%)] & $1(0,3 \%)$ & 0 & $1(0,3 \%)$ & $1(1,4 \%)$ & $2(0,5 \%)$ \\
\hline $\begin{array}{l}\text { EHQ Total (mean } \pm \\
\text { sd; median, [IQR]) }\end{array}$ & $\begin{array}{l}40.1 \pm 8.59 ; 39 \\
\quad[34-45]\end{array}$ & $\begin{array}{c}50.4 \pm 9.24 ; 49 \\
{[43.8-58.2]}\end{array}$ & $41 \pm 9.09 ; 39,[34-46]$ & $\begin{array}{l}38.7 \pm 7.4 ; 38,[33.8- \\
\quad 43.2]\end{array}$ & $\begin{array}{l}40.5 \pm 8.82 ; 39,[34- \\
\quad 46]\end{array}$ \\
\hline EHQ knowledge & $\begin{array}{l}12.7 \pm 3.21 ; 13 \\
{[10-15]}\end{array}$ & $\begin{array}{l}14.1 \pm 2.8 ; 14,[13- \\
15.2]\end{array}$ & $\begin{array}{l}12.8 \pm 3.2 ; 13,[10.2- \\
15]\end{array}$ & $\begin{array}{l}12.5 \pm 3.15 ; 13.5 \\
{[10-15]}\end{array}$ & $\begin{array}{l}12.7 \pm 3.19 ; 13,[10- \\
15]\end{array}$ \\
\hline EHQ feelings & $10 \pm 2.59 ; 10,[8-12]$ & $\begin{array}{l}11.4 \pm 2.08 ; 11 \\
{[10-13]}\end{array}$ & $\begin{array}{l}10.1 \pm 2.57 ; 10 \\
{[9-12]}\end{array}$ & $\begin{array}{l}9.78 \pm 2.41 ; 10 \\
\quad[8-12]\end{array}$ & $10.1 \pm 2.54 ; 10,[9-12]$ \\
\hline EHQ problems & $\begin{array}{l}17.4 \pm 5 ; 16,[14- \\
19.8]\end{array}$ & $\begin{array}{l}24.9 \pm 7.18 ; 24.5 \\
{[18.8-31]}\end{array}$ & $\begin{array}{l}18.0 \pm 5.6 ; 16,[14- \\
21]\end{array}$ & $\begin{array}{l}16.4 \pm 3.69 ; 16 \\
{[14-18]}\end{array}$ & $\begin{array}{l}17.7 \pm 5.31 ; 16,[14- \\
20]\end{array}$ \\
\hline Drive for thinness & $\begin{array}{l}8.1 \pm 7.09 ; 6,[2.25- \\
12]\end{array}$ & $\begin{array}{l}16 \pm 7.84 ; 16.5 \\
{[10.8-23]}\end{array}$ & $8.77 \pm 7.47 ; 6,[3-13]$ & $4 \pm 3.94 ; 3,[1-5]$ & $7.81 \pm 7.17 ; 5,[2-12]$ \\
\hline Vegans [No. (\%)] & $3(1,1 \%)$ & $2(8,3 \%)$ & $5(1,7 \%)$ & 0 & $5(1,4 \%)$ \\
\hline Vegetarians [No. $(\%)]$ & $16(6,1 \%)$ & $3(12,5 \%)$ & $19(6,6 \%)$ & $1(1,4 \%)$ & $20(5,5 \%)$ \\
\hline $\begin{array}{l}\text { With anorexia nervosa } \\
\text { [No. }(\%)]\end{array}$ & // & // & $18(6,3 \%)$ & 0 & $18(5 \%)$ \\
\hline $\begin{array}{l}\text { With bulimia nervosa } \\
\text { [No. }(\%)]\end{array}$ & // & // & $7(2,4 \%)$ & 0 & $7(1,9 \%)$ \\
\hline $\begin{array}{l}\text { With a physical ill- } \\
\text { ness/disease/disor- } \\
\text { der [No. }(\%)]\end{array}$ & $19(7,2 \%)$ & $5(20,8 \%)$ & $42(14,6 \%)$ & $6(8,3 \%)$ & $48(13,4 \%)$ \\
\hline $\begin{array}{l}\text { Followed at least one } \\
\text { diet }[\text { No. }(\%)]\end{array}$ & $124(47,3 \%)$ & $21(87,5 \%)$ & $145(50,7 \%)$ & $27(37,5 \%)$ & $172(48,8 \%)$ \\
\hline
\end{tabular}

No male participants reported an eating disorder history. Participants with a BMI lower than 18 have underweight. Participants with a BMI higher than 25.0 but lower than 30.0 are considered to have overweight. Participants with a BMI equal to or higher than 30 have at least Grade I Obesity

$E D$ eating disorder(s), $E H Q$ eating habits questionnaire, $I Q R$ interquartile range

having followed at least one diet in their lifetime $(\beta=0.07$ CI 95\% (0.03-0.12), $p<0.001)$. In addition, for this subscale, for every 10 points increase of BMI, the score was reduced by $\beta=-0.11$ (CI 95\% -0.18 to $-0.03, p<0.01$ ).

Similar to the Problems subscale, also the Knowledge subscale scores were influenced by commitment to vegetarian diets (Fig. 1C and Appendix table 3. $\beta=0.08$ CI 95\% $(0.0-0.16), p<0.05)$ and non-significantly by vegan ones ( $\beta=0.16$ CI 95\% $(-0.01-0.32), p=0.067)$. Participants who followed at least one diet in their lifetime scored higher at this subscale $(\beta=0.08$ CI 95\% $(0.05-0.12), p<0.001)$. The negative impact of these factors was to some extent mitigated by the effect of BMI: for every ten units of BMI, the score was reduced by a factor of $\beta=-0.12$, (CI 95\% -0.18 to -0.06$), p<0.001$.

Considering the EHQ Total score, the factors that influenced this score overlapped with those influencing the Problem subscale (Fig. 1D and Appendix Table 4). Commitment to a vegetarian $(\beta=0.08$, CI 95\% $(0.04-0.13), p<0.001)$ or vegan dietary regimen $(\beta=0.15$, CI $95 \%(0.06-0.25)$, $p<0.01)$, or having followed at least $1 \operatorname{diet}(\beta=0.1$, CI 95\% $(0.08-0.12), p<0.001)$ were factors that increased the severity of manifestations of orthorexia. Moreover, a history of a physical illness, disease, or disorder $(\beta=0.04$, CI 95\% $(0.02-0.07), p<0.01)$ also increased the symptomatology, but not to the same extent as having suffered, or is suffering from $\mathrm{AN}(\beta=0.17$, CI 95\% (0.13-0.22), $p<0.001)$. For 
A

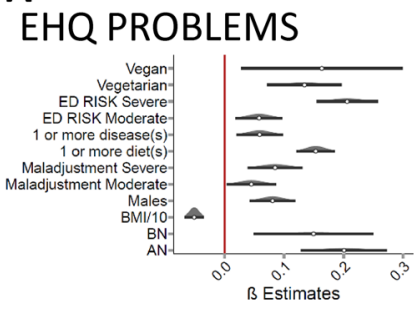

D

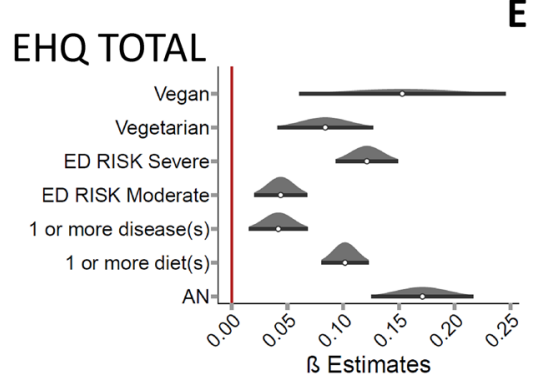

Fig. 1 Factors associated with features of orthorexia nervosa. $\mathrm{EHQ}=$ eating habits questionnaire; ED RISK = classes of symptoms severity; Maladjustment $=$ classes of symptoms severity BMI = body mass index; $\mathrm{BN}=$ bulimia nervosa; $\mathrm{AN}=$ anorexia nervosa. The red line represents the significance level. Factors at the left of the

the EHQ Total score, we report a significant association of the score with the severity of eating disorder symptoms as measured by the ED Risk subscale (moderate symptoms: $\beta=0.04$, CI 95\% (0.02-0.07), $p<0.001$; severe symptoms: $\beta=0.12$, CI 95\% (0.09-0.15), $p<0.001)$ ).

\section{ON, diets and ED characteristics in the high orthorexic sample}

We defined different binomial regression models to evidence which factors increased the odds of experiencing clinically relevant manifestations of orthorexia (defined as an EHQ Total score $\geq 50$; Fig. 1E, Appendix Table 5). As previously described for the EHQ Total score, the severity of eating disorder symptoms (measured with ED Risk subscale) was associated with a higher risk of experiencing relevant manifestations of ON (moderate symptoms: Odds Ratio $=2.07$, CI 95\% (1.13-3.8), $p<0.05$; severe symptoms: $\mathrm{OR}=4.52$, CI 95\% (2.39-8.54), $p<0.001)$ ), a risk also conveyed by having followed at least one diet in the lifetime $(\mathrm{OR}=4.64$, CI 95\% (2.87-7.47), $p<0.001$ ), or reporting food avoidance, irrespective of the reason $(\mathrm{OR}=2.84$, CI 95\% (1.93-4.18), $p<0.001)$. Have a physical illness, disease, or disorder was also associated with higher odds of experiencing relevant manifestations (OR $=1.75$, CI 95\% (1.09-2.81), $p<0.05)$, but a history of anorexia nervosa was the factor that increased the most the risk of experiencing relevant manifestations of orthorexia (OR $=7.55$, CI 95\% (3.24-17.62),

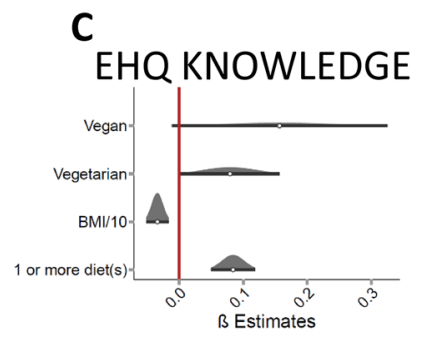

BINOMIAL

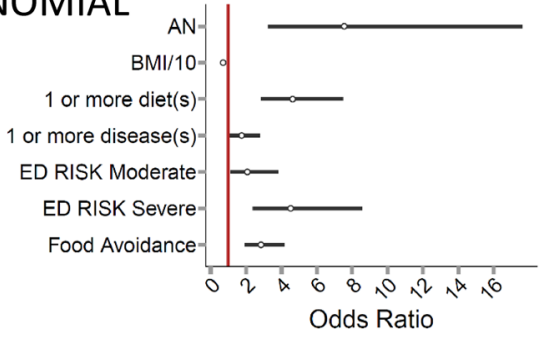

line are associated with a lower score, whereas factors at the right increase the score. Factors that were significantly associated with a higher score at the AEHQ Problems subscale. B EHQ Feelings subscale. CEHQ Knowledge subscale. D EHQtotal score. E Factors that increase the odds of having relevant features of orthorexia nervosa

$p<0.001)$. The only protective factor was BMI. For every ten units of BMI, OR $=0.3$, CI 95\% (0.14-0.64), $p<0.01)$.

\section{ON and ED characteristics in the sample with at least one diet}

To further investigate the role of dieting, we analysed characterised different variables related to participants' nutrition and dietetic choices. At t-zero, approximately half (48\%, Table 1) of our sample with at least one diet in the lifetime.

Participants who reported having followed more than ten diets had higher EHQ Problems scores $(\beta=0.19$, CI 95\% $(0.13-0.24), \mathrm{p}<0.001)$ than participants who had followed less than ten diets (Fig. 2A, Appendix Table 6). Moreover, we found that participants who had followed a diet more than a month before taking the survey experienced fewer manifestations than those who followed a diet in the last month ( $\beta=-0.13$, CI 95\% ( $-0.17--0.09), p<0.001)$. Commitment to a vegetarian or vegan diet and higher EDs symptom severity further increased the EHQ Problems score (Appendix Table 6).

Analogously, we found that having followed a diet more than a month before taking the survey resulted in a lower EHQ Feelings score than a more recent diet $(\beta=-0.12$, CI $95 \%$ ( -0.18 to -0.05$), p<0.001)$. Moreover, for every 10-point increase in the BMI, the score was reduced by a factor $\beta=-0.15$ (CI 95\% ( -0.24 to -0.05$)$ ), $p<0.01$ ), whereas the only other variable that significantly increased the manifestations measured by this subscale was the eating 
Fig. 2 Orthorexia nervosa in participants with one diet in their lifetime. $\mathrm{EHQ}=$ eating habits questionnaire; ED RISK $=$ classes of symptoms severity; $\mathrm{BMI}=$ body mass index; $\mathrm{AN}=$ anorexia nervosa. A Factors that were significantly associated with a higher score at the EHQ Problems subscale. $\mathbf{B}$ at the EHQ Feelings subscale. $\mathbf{C}$ at the EHQ Knowledge subscale. D at the EHQ total score
A

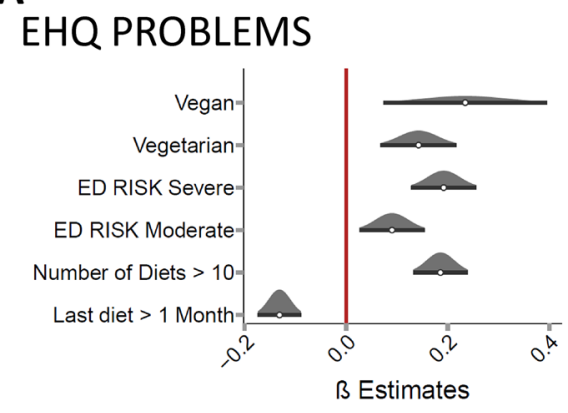

C

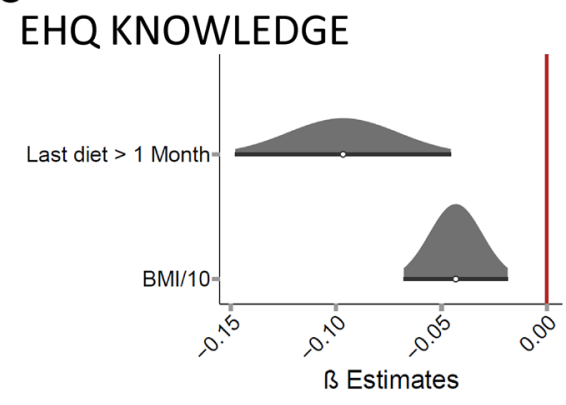

B

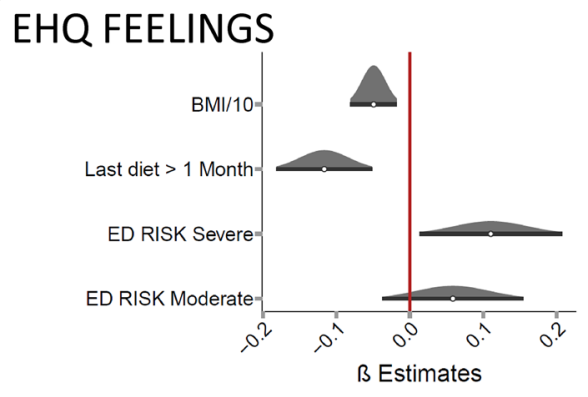

D

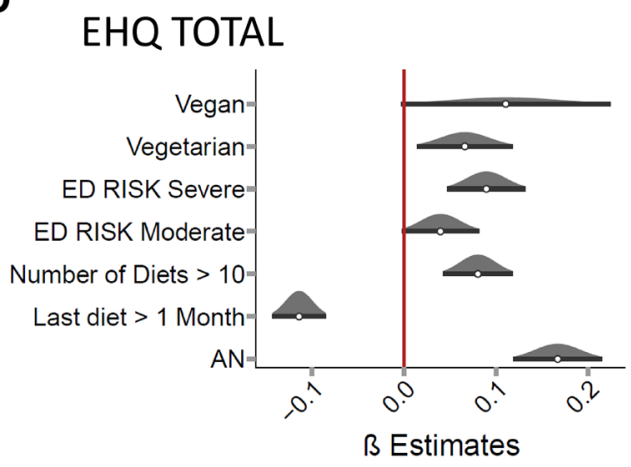

disorders symptoms severity (moderate: not significant, severe symptoms: $\beta=0.11$, CI $95 \%(0.01-0.21), p<0.05$, Fig. 2B, Appendix Table 7A. See Appendix Table 7B for model characteristics when also considering item 9 in the Feelings score).

For the participants who reported having followed at least one diet, the characteristics measured by the EHQ Knowledge subscale could only be mitigated by two factors: BMI (for every ten units: $\beta=-0.13$, CI $95 \%(-0.2$ to -0.06 ), $p<0.001)$ and, as reported for the previous subscales, the time from the last diet (at least 1 month from the survey $\beta=-0.1$, CI 95\% ( -0.15 to -0.05$), p<0.001$, Fig. 2 C, Appendix Table 8).

Regarding the characteristics of orthorexia measured by the EHQ Total score, participants who had followed more than ten diets in their lifetime reported higher scores than those who followed a smaller number of diets $(\beta=0.08$, CI 95\% (0.04-0.12), $p<0.001$, Fig. 2D, Appendix Table 9). On the other hand, if the last followed diet dated to more than a month before the survey, the manifestations were somehow mitigated ( $\beta=-0.11$, CI 95\% ( -0.14 to -0.09$), \mathrm{p}<0.001$ ). Commitment to a vegetarian regimen further increased the EHQ Total score ( $\beta=0.07$, CI 95\% (0.01-0.12), $p<0.001)$, whereas being vegan did not reach statistical significance. Participants who experienced severe symptoms of eating disorders (measured with ED Risk subscale) were also more likely to experience more manifestations of orthorexia $(\beta=0.09$, CI 95\% (0.05-0.13), $p<0.001$, whereas moderate symptoms did not significantly influence the EHQ
Total score). Participants who reported a history of AN also reported worse manifestations of orthorexia $(\beta=0.17$, CI $95 \%(0.12-0.22), p<0.001)$. Finally, we tested if this group of participants (who followed at least one diet in their lifetime) reported EHQ scores 6 months after the first survey completion that could be predicted with variables acquired at t-zero. However, the EHQ scores of this sub-sample could be best described by the same models reported for the overall sample (i.e., models considering the EHQ scores at t-zero).

\section{ON characteristics at 6 months}

Six months after the first cross-sectional survey, participants were automatically re-contacted via email. A total of 358 participants $(33,3 \%$ of the sample size of the first cross-sectional) agreed to participate in a second wave and completed all the questionnaires. We tested all the variables acquired at the first cross-sectional in predicting the EHQ scores 6 months later.

The best model to predict the EHQ Total score relies on the EHQ Total score itself and the drive for thinness at t-zero (Fig. 3A. For each point at the EHQ Total score at t-zero: $\beta=0.02$, CI 95\% (0.01-0.02), $p<0.001$; for each point at the drive for thinness $\beta=0.003$ CI 95\% (0.00-0.01), $p<0.01$; Marginal $\mathrm{R}^{2}$ of the model $=0.53$ ).

Similarly, the EHQ Problems subscale score at 6 months is predicted by the EHQ Problems score and drive for thinness at t-zero (Fig. 3B. EHQ Problems score at t-zero: 
Fig. 3 Predictors of Orthorexia Nervosa after 6 months. $\mathrm{EHQ}=$ Eating Habits Questionnaire; EHQ Total score (panel A)), EHQ Problems (panel B)), C Simple regression model of the EHQ Knowledge subscale D Simple regression model of the EHQ Feelings subscale
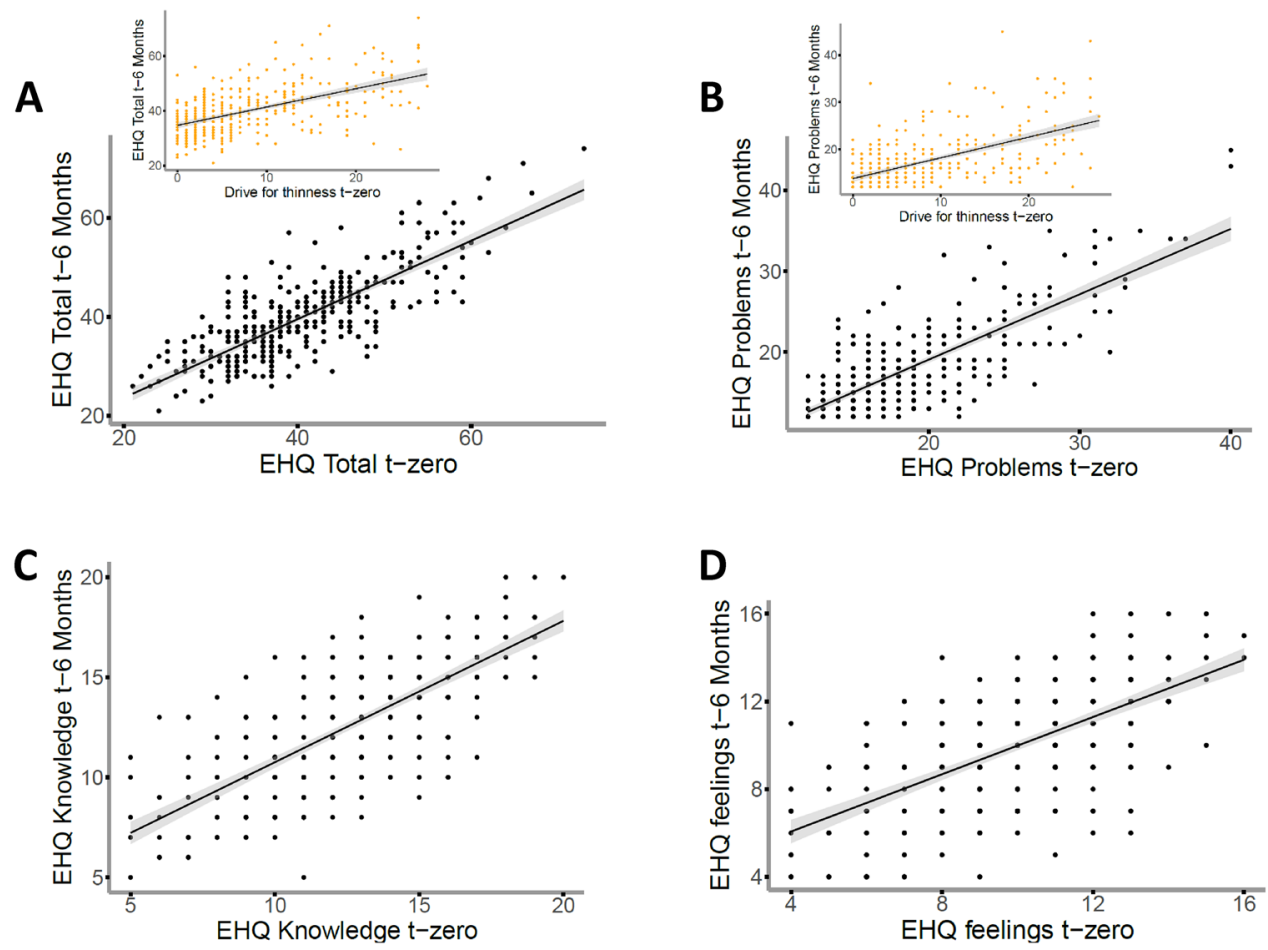

$\beta=0.04$, CI 95\% (0.03-0.04), $p<0.001$; for each point at the drive for thinness $\beta=0.01$ CI 95\% (0.00-0.01), $p<0.01$; Marginal $\mathrm{R}^{2}$ of the model $=0.45$ ).

On the other hand, the EHQ Knowledge and EHQ Feelings scores can be best described by considering solely the Knowledge and Feelings scores at t-zero, respectively (Fig. 3C, D). EHQ Knowledge score at t-zero: $\beta=0.06$, CI $95 \%(0.05-0.07), p<0.001$, Marginal $\mathrm{R}^{2}$ of the model $=0.30$; EHQ Feelings score at t-zero: $\beta=0.07$ CI $95 \%$ $(0.05-0.08), p<0.001$; Marginal $\mathrm{R}^{2}$ of the model $\left.=0.23\right)$.

\section{Discussion}

It is of high clinical relevance to ascertain whether $\mathrm{ON}$ is a diagnostic category distinct from other eating disorders but characterized by specific dysfunctions in food-related behaviors. The current study aimed at extending our knowledge on $\mathrm{ON}$ by investigating its relationships with the amount and types of diets undertaken through a cross-sectional and longitudinal investigation. Dietary restrictions can be considered EDs' risk factors [79] and are central elements in the ON hypothesis as a diagnostic category within eating disorders.

Our cross-sectional results have highlighted how the ON is dependent not only on the type (vegan and vegetarian) $[13,24]$ but also on the frequency of diet: having followed at least one diet in the lifetime is associated with more severe
ON manifestations. Furthermore, as reported in other studies $[34,35,80]$, our results confirm how the ON is related to the severity of EDs symptomatology linked to the dissatisfaction of one's body and the ideal of thinness, and having had a diagnosis of anorexia nervosa.

Impairment of ON in daily life, based on the EHQ Problems score, was also associated with the type and number of diets, EDs, physical symptoms, and male gender. Even though none of the males reported a previous diagnosis of EDs, these results had been reported by other studies [81, 82]. The increasing focus of males on healthy lifestyles and diets could explain these data. Congruently, the knowledge about healthy diets was associated with dieting and diet type (i.e., vegan and vegetarian). The emotions about dieting were associated with dysfunctional beliefs on thinness and body dissatisfaction. Furthermore, our results show that higher BMI scores could be considered a protective factor from ON features: for every 10 points in the BMI, the EHQ score is 10 to $20 \%$ lower. Data could be interpreted as individuals with a lower BMI being more prone to dieting and weight control while also experiencing more significant concern and discomfort, impacting their global functioning. Overall, results confirm that $\mathrm{ON}$ is closely linked to AN's presence in the past [16] and that pursuing diets and low BMI can be prodromes for eating problems.

The results obtained by considering the individuals with relevant orthorexic manifestations (EHQ $>90$ th percentile) further support our data: features related to EDs, diets, and diseases are significantly associated with relevant ON 
scores, whereas a higher BMI is a protective factor. While we expected a diagnosis of ED (including AN) to be associated with higher ON manifestations [36], having followed at least one diet during the lifetime (for any reason) and having EDs features (without an overt diagnosis) is equally associated with relevant $\mathrm{ON}$ characteristics. In this context, data could be due to participants having had EDs in the past or being at risk of developing EDs. Still, this piece of evidence might also point at the overlap of ON with body dissatisfaction, drive for thinness, and bulimic behaviors following several dietary restrictions.

Regarding participants who followed at least one diet in the past, our results showed that the number $(>10)$ and type of diet increase the ON manifestations and its consequences (Problems subscale). On the other hand, having stopped the diet for more than a month is considered a protective factor for $\mathrm{ON}$; in this context, the presence of a higher BMI continues to be a protective factor. Participants following continuous dietary regimes may be at greater risk of developing ON and social and interpersonal problems resulting from dieting. Thus, it becomes pivotal to identify the number of diets conducted in the past and time elapsed from the last diet, considering the beneficial role of discontinuing diet on reducing $\mathrm{ON}$.

Orthorexic features that emerged during the first wave were the best predictor of the ON characteristics after 6 months. Therefore, the orthorexic features do not change and are stable in time, supporting the hypothesis that $\mathrm{ON}$ can be considered a construct independent from EDs. The motivations of thinness represent the second-best predictor of $\mathrm{ON}$ : participants who endorse dysfunctional thinness ideals are more likely to experience psychological and interpersonal problems 6 months later.

No relationship was found with characteristics related to anxiety, depression, or obsessive and compulsive symptoms, confirming a part of the literature about the independence of the $\mathrm{ON}$ from these constructs [83-85].

\section{Conclusion}

Our results confirm that $\mathrm{ON}$ is more represented in individuals with a past diagnosis of EDs [16], in individuals who pursue a restrictive diet or a vegan, vegetarian one $[16$, 22]. In addition, we have found that the number of diets, beliefs, and behaviors related to the ideals of thinness or body dissatisfaction are common features of ON. Moreover, considering that $\mathrm{ON}$ features assessed at $\mathrm{t}$-zero are the best ON predictors after 6 months, we can presume that the ON construct is stable over time. All these aspects characterise the $\mathrm{ON}$ as a multi-faceted construct that could be considered a risk factor for the development or maintenance of EDs or could represent a problem per se, considering that $\mathrm{ON}$ is related to social or interpersonal impairments that remain constant time. Healthcare professionals should consider the dieting frequency during the lifetime as a possible link with the $\mathrm{ON}$ and with its problematic consequences. Moreover, it would be helpful to also consider orthorexic features in the assessment of the EDs.

\section{Strengths and limits}

Regrettably, we have no information about the onset or duration of the physical or dietary problem, nor whether it has been resolved at the moment of questionnaire administration. Another limit concerns the second wave: about $1 / 3$ of the individuals agreed to follow-up at 6 months. Even though there were no substantial differences in the demographic and psychological features between those who participated once or twice, the prospective evidence presented herein has to be taken with caution and confirmed with further studies. Moreover, the sample was composed of non-clinical Italian students and was mostly female: this poses an issue about the generalizability of our results. A wide array of validated questionnaires has been used to ascertain ON's main contributors and benchmarks, but the assessment was based only on self-report questionnaires that ignore ego-syntonic characteristics. Furthermore, the EHQ does not assess negative emotions and concerns about eating unhealthy foods. Future studies should consider interviews or clinical judgments to overcome these problems.

\section{What is already known on the subject?}

Orthorexia Nervosa (ON) is an unhealthy eating behavior characterised by an excessive concern for a healthy diet or food quality. Much research to date has highlighted a significant intersection between ON and ED. Still, among the distinctions, there is the dichotomy quantity-versus-quality of food intake that needs more investigation.

\section{What does this study add?}

The number of diets, beliefs, and behaviors related to the ideals of thinness or body dissatisfaction are common features of ON. Moreover, considering that having ON features in the past is the best ON predictor in the present, we can presume that $\mathrm{ON}$ is a construct stable over time.

Supplementary Information The online version contains supplementary material available at https://doi.org/10.1007/s40519-021-01353-8.

Acknowledgements We thank Prof. Aram Megighian and Dr Giulia Fattorini for their help with participant recruitment.

Author contributions Caterina Novara and Susanna Pardini conceptualised the study. All of the authors contributed to the manuscript's 
methodology, review, and editing phases and approved the article's final version. Caterina Novara and Nicola Meda supervised the data collection. Nicola Meda conducted the statistical analysis and the visualisations.

Funding This work was carried out within the framework of the project "Use-inspired basic research," for which the Department of General Psychology of the University of Padova has been recognised as "Dipartimento di Eccellenza" (Department of Excellence) by the Ministry of University and Research.

Data availability The datasets used and analysed for the present study will be provided by the corresponding author upon reasonable request.

\section{Declarations}

Conflict of interest The authors declare that they have no conflict of interest.

Ethical approval Participants were fully informed about the research's aim, and reported written consent was obtained from all participants. They were invited to complete a series of self-report questionnaires. The anonymity and the confidentiality of the collected data were guaranteed. This study was conducted according to the Declaration of Helsinki and was approved by the Department of General Psychology Ethical Committee (Area 17) of the University of Padova.

Open Access This article is licensed under a Creative Commons Attribution 4.0 International License, which permits use, sharing, adaptation, distribution and reproduction in any medium or format, as long as you give appropriate credit to the original author(s) and the source, provide a link to the Creative Commons licence, and indicate if changes were made. The images or other third party material in this article are included in the article's Creative Commons licence, unless indicated otherwise in a credit line to the material. If material is not included in the article's Creative Commons licence and your intended use is not permitted by statutory regulation or exceeds the permitted use, you will need to obtain permission directly from the copyright holder. To view a copy of this licence, visit http://creativecommons.org/licenses/by/4.0/.

\section{References}

1. Oberle CD, Samaghabadi RO, Hughes EM (2017) Orthorexia nervosa: assessment and correlates with gender, BMI, and personality. Appetite 108:303-310

2. Moroze RM, Dunn TM, Craig Holland J, Yager J, Weintraub $P$ (2015) Microthinking about micronutrients: a case of transition from obsessions about healthy eating to near-fatal "orthorexia nervosa" and proposed diagnostic criteria. Psychosomatics 56:397-403

3. Dunn TM, Bratman S (2016) On orthorexia nervosa: a review of the literature and proposed diagnostic criteria. Eat Behav 21:11-17

4. Valente M, Syurina EV, Donini LM (2019) Shedding light upon various tools to assess orthorexia nervosa: a critical literature review with a systematic search. Eat Weight Disord-Stud Anorex, Bulim Obes 24(4):671-682. https://doi.org/10.1007/ s40519-019-00735-3

5. Opitz MC, Newman E, Alvarado Vázquez Mellado AS, Robertson MKDA, Sharpe H (2020) The psychometric properties of orthorexia nervosa assessment scales: a systematic review and reliability generalization. Appetite 155:104797. https://doi.org/ 10.1016/j.appet.2020.104797

6. Cena H, Barthels F, Cuzzolaro M, Bratman S, Brytek-Matera A, Dunn T et al (2019) Definition and diagnostic criteria for orthorexia nervosa: a narrative review of the literature. Eat Weight Disord - Stud Anorex Bulim Obes 24:209-246

7. Dunn TM, Gibbs J, Whitney N et al (2017) Prevalence of orthorexia nervosa is less than $1 \%$ : data from a US sample. Eat Weight Disord - Stud Anorex Bulim Obes 22:185-192. https://doi.org/10. 1007/s40519-016-0258-8

8. Missbach B, Dunn TM, Konig JS (2016) We need new tools to assess orthorexia nervosa. A commentary on "prevalence of orthorexia nervosa among college students based on Bratman's test and associated tendencies.” Appetite. https://doi.org/10.1016/j. appet.2016.07.010

9. Håman L, Barker-Ruchti N, Patriksson G, Lindgren E-C (2015) Orthorexia nervosa: an integrative literature review of a lifestyle syndrome. Int J Qual Stud Health Well-Being 10:26799

10. Barthels F, Barrada JR, Roncero M (2019) Orthorexia nervosa and healthy orthorexia as new eating styles. PLoS ONE 14:e0219609

11. Cheshire A, Berry M, Fixsen A (2020) What are the key features of orthorexia nervosa and influences on its development? A qualitative investigation. Appetite 155:104798

12. Koven NS, Abry AW (2015) The clinical basis of orthorexia nervosa: emerging perspectives. Neuropsychiatr Dis Treat 11:385-394

13. Barthels F, Meyer F, Pietrowsky R (2018) Orthorexic and restrained eating behaviour in vegans, vegetarians, and individuals on a diet. Eat Weight Disord - Stud Anorex Bulim Obes 23:159-166

14. Park SW, Kim JY, Go GJ, Jeon ES, Pyo HJ, Kwon YJ (2011) Orthorexia nervosa with hyponatremia, subcutaneous emphysema, pneumomediastimum, pneumothorax, and pancytopenia. Electrolytes Blood Press 9:32

15. Novara C, Maggio E, Piasentin S, Pardini S, Mattioli S (2021) Orthorexia nervosa: differences between clinical and non-clinical samples. BMC Psychiatry 21:341

16. Segura-Garcia C, Ramacciotti C, Rania M, Aloi M, Caroleo M, Bruni A et al (2015) The prevalence of orthorexia nervosa among eating disorder patients after treatment. Eat Weight Disord - Stud Anorex Bulim Obes 20:161-166

17. Kinzl JF, Hauer K, Traweger C, Kiefer I (2006) Orthorexia nervosa in dieticians. Psychother Psychosom 75:395-396

18. Bağcı Bosi AT, Çamur D, Güler Ç (2007) Prevalence of orthorexia nervosa in resident medical doctors in the faculty of medicine (Ankara, Turkey). Appetite 49:661-666

19. Agopyan A, Kenger EB, Kermen S, Ulker MT, Uzsoy MA, Yetgin MK (2019) The relationship between orthorexia nervosa and body composition in female students of the nutrition and dietetics department. Eat Weight Disord - Stud Anorex Bulim Obes 24:257-266

20. Korinth A, Schiess S, Westenhoefer J (2010) Eating behaviour and eating disorders in students of nutrition sciences. Public Health Nutr 13:32-37

21. Bo S, Zoccali R, Ponzo V, Soldati L, De Carli L, Benso A et al (2014) University courses, eating problems and muscle dysmorphia: are there any associations? J Transl Med 12:221

22. Brytek-Matera A (2021) Vegetarian diet and orthorexia nervosa: a review of the literature. Eat Weight Disord - Stud Anorex Bulim Obes 26:1-11

23. Donini LM, Marsili D, Graziani MP, Imbriale M, Cannella C (2004) Orthorexia nervosa: a preliminary study with a proposal for diagnosis and an attempt to measure the dimension of the phenomenon. Eat Weight Disord - Stud Anorex Bulim Obes 9:151-157 
24. Zickgraf HF, Ellis JM, Essayli JH (2019) Disentangling orthorexia nervosa from healthy eating and other eating disorder symptoms: relationships with clinical impairment, comorbidity, and selfreported food choices. Appetite 134:40-49

25. Bratman S (2017) Orthorexia vs. theories of healthy eating. Eat Weight Disord - Stud Anorex Bulim Obes. 22:381-385

26. Dunn TM, Gibbs J, Whitney N, Starosta A (2017) Prevalence of orthorexia nervosa is less than $1 \%$ : data from a US sample. Eat Weight Disord - Stud Anorex Bulim Obes 22:185-192

27. Fidan T, Ertekin V, Işikay S, Kırpınar I (2010) Prevalence of orthorexia among medical students in Erzurum. Turkey Compr Psychiatry 51:49-54

28. Dell'Osso L, Abelli M, Carpita B, Massimetti G, Pini S, Rivetti L et al (2016) Orthorexia nervosa in a sample of Italian university population. Riv Psichiatr. https://doi.org/10.1708/2476.25888

29. Segura-García C, Ammendolia A, Procopio L, Papaianni MC, Sinopoli F, Bianco C et al (2010) Body uneasiness, eating disorders, and muscle dysmorphia in individuals who overexercise. $\mathrm{J}$ Strength Cond Res 24:3098-3104

30. Varga M, Thege BK, Dukay-Szabó S, Túry F, van Furth EF (2014) When eating healthy is not healthy: orthorexia nervosa and its measurement with the ORTO-15 in Hungary. BMC Psychiatry $14: 59$

31. Bundros J, Clifford D, Silliman K, Neyman MM (2016) Prevalence of orthorexia nervosa among college students based on Bratman's test and associated tendencies. Appetite 101:86-94

32. Depa J, Schweizer J, Bekers S-K, Hilzendegen C, Stroebele-Benschop N (2017) Prevalence and predictors of orthorexia nervosa among German students using the 21-item-DOS. Eat Weight Disord - Stud Anorex Bulim Obes 22:193-199

33. Reynolds R (2018) Is the prevalence of orthorexia nervosa in an Australian university population 6.5\%? Eat Weight Disord - Stud Anorex Bulim Obes. 23:453-458

34. Missbach B, Hinterbuchinger B, Dreiseitl V, Zellhofer S, Kurz C, König J (2015) When eating right, is measured wrong! a validation and critical examination of the ORTO-15 questionnaire in German. PLoS ONE 10:e0135772

35. Parra-Fernández M-L, Rodríguez-Cano T, Onieva-Zafra M-D, Perez-Haro MJ, Casero-Alonso V, Fernández-Martinez E et al (2018) Prevalence of orthorexia nervosa in university students and its relationship with psychopathological aspects of eating behaviour disorders. BMC Psychiatry 18:364

36. Roncero M, Barrada JR, Perpiñá C (2017) Measuring orthorexia nervosa: psychometric limitations of the ORTO-15. Span J Psychol 20:E41

37. Sanlier N, Yassibas E, Bilici S, Sahin G, Celik B (2016) Does the rise in eating disorders lead to increasing risk of orthorexia nervosa? Correlations with gender, education, and body mass index. Ecol Food Nutr 55:266-278

38. McComb SE, Mills JS (2019) Orthorexia nervosa: a review of psychosocial risk factors. Appetite 140:50-75

39. Karakuş B (2017) Orthorexia nervosa trends among students of nutrition and dietetics department at a university in Istanbul. North Clin Istanb. https://doi.org/10.14744/nci.2017.20082

40. Brytek-Matera A, Donini LM, Krupa M, Poggiogalle E, Hay P (2015) Orthorexia nervosa and self-attitudinal aspects of body image in female and male university students. J Eat Disord 3:2

41. Brytek-Matera A, Fonte ML, Poggiogalle E, Donini LM, Cena H (2017) Orthorexia nervosa: relationship with obsessive-compulsive symptoms, disordered eating patterns and body uneasiness among Italian university students. Eat Weight Disord - Stud Anorex Bulim Obes 22:609-617

42. Luck-Sikorski C, Jung F, Schlosser K, Riedel-Heller SG (2019) Is orthorexic behavior common in the general public? A large representative study in Germany. Eat Weight Disord - Stud Anorex Bulim Obes 24:267-273
43. Almeida C, Vieira Borba V, Santos L (2018) Orthorexia nervosa in a sample of Portuguese fitness participants. Eat Weight Disord - Stud Anorex Bulim Obes 23:443-451

44. Herranz Valera J, Acuña Ruiz P, Romero Valdespino B, Visioli F (2014) Prevalence of orthorexia nervosa among ashtanga yoga practitioners: a pilot study. Eat Weight Disord - Stud Anorex Bulim Obes 19:469-472

45. Oberle CD, Lipschuetz SL (2018) Orthorexia symptoms correlate with perceived muscularity and body fat, not BMI. Eat Weight Disord - Stud Anorex Bulim Obes 23:363-368

46. Barrada JR, Roncero M (2018) Bidimensional structure of the orthorexia: development and initial validation of a new instrument. An Psicol 34:283

47. Dell'Osso L, Carpita B, Muti D, Cremone IM, Massimetti G, Diadema E et al (2018) Prevalence and characteristics of orthorexia nervosa in a sample of university students in Italy. Eat Weight Disord - Stud Anorex Bulim Obes 23:55-65

48. Asil E, Sürücüoğlu MS (2015) Orthorexia nervosa in Turkish dietitians. Ecol Food Nutr 54:303-313

49. Hyrnik J, Janas-Kozik M, Stochel M, Jelonek I, Siwiec A, Rybakowski JK (2016) The assessment of orthorexia nervosa among 1899 polish adolescents using the ORTO-15 questionnaire. Int J Psychiatry Clin Pract 20:199-203

50. Brytek-Matera A, Czepczor-Bernat K, Olejniczak D (2018) Foodrelated behaviours among individuals with overweight/obesity and normal body weight. Nutr J 17:93

51. Tremelling K, Sandon L, Vega GL, McAdams CJ (2017) Orthorexia nervosa and eating disorder symptoms in registered dietitian nutritionists in the United States. J Acad Nutr Diet 117:1612-1617

52. Gleaves DH, Graham EC, Ambwani S (2013) Measuring, "orthorexia": development of the eating habits questionnaire. Int J Educ Psychol Assess 12:1-18

53. Depa J, Barrada J, Roncero M (2019) Are the motives for food choices different in orthorexia nervosa and healthy orthorexia? Nutrients 11:697

54. Catalina Zamora ML, Bote Bonaechea B, García Sánchez F, Ríos Rial B (2005) Orthorexia nervosa. A new eating behavior disorder? Actas Esp Psiquiatr 33:66-68

55. Missbach B, Barthels F (2017) Orthorexia nervosa: moving forward in the field. Eat Weight Disord - Stud Anorex Bulim Obes 22:1-1

56. Meda N, Pardini S, Slongo I, Bodini L, Zordan MA, Rigobello P et al (2021) Students' mental health problems before, during, and after COVID-19 lockdown in Italy. J Psychiatr Res 134:69-77

57. Harris PA, Taylor R, Thielke R, Payne J, Gonzalez N, Conde JG (2009) Research electronic data capture (REDCap) — a metadatadriven methodology and workflow process for providing translational research informatics support. J Biomed Inform 42:377-381

58. Beck AT, Steer RA, Brown GK (1996) Manual for the Beck Depression Inventory-II. TX Psychological Corporation, San Antonio, TX. https://doi.org/10.1037/t00742-000

59. Sica C, Ghisi M (2007) The Italian versions of the Beck anxiety inventory and the Beck depression inventory-II: psychometric properties and discriminant power. Leading-edge psychological tests and testing research. Nova Science Publishers, Hauppauge, NY, US, pp 27-50

60. Ghisi M, Flebus G, Montano A, Sanavio E, Sica C (2006) Beck depression inventory-second edition. Adattamento italiano: Manuale. Firenze: Giunti O-S Organizzazioni Speciali

61. Wang YP, Gorenstein C (2013) Psychometric properties of the Beck depression inventory-II: a comprehensive review. Braz J Psychiatry 35(4):416-431. https://doi.org/10.1590/1516-44462012-1048 (Epub 2013 Dec 23. PMID: 24402217)

62. Creamer M, Foran J, Bell R (1995) The Beck anxiety inventory in a non-clinical sample. Behav Res Ther 33:477-485 
63. Steer RA, Beck AT (1997) Beck anxiety inventory. Evaluating stress: a book of resources. Scarecrow Education, Lanham, MD, US, pp 23-40

64. Beck AT, Epstein N, Brown G, Steer RA (1988) An inventory for measuring clinical anxiety: psychometric properties. J Consult Clin Psychol 56:893-897

65. Sica C, Coradeschi D, Ghisi M, Sanavio E (2006) Beck anxiety inventory. Adattamento italiano: Manuale. Firenze: Giunti O.S. Organizzazioni Speciali

66. Abramowitz JS, Deacon BJ (2006) Psychometric properties and construct validity of the obsessive-compulsive inventoryrevised: replication and extension with a clinical sample. J Anxiety Disord 20:1016-1035

67. Sica C, Ghisi M, Altoè G, Chiri LR, Franceschini S, Coradeschi D et al (2009) The Italian version of the obsessive compulsive inventory: its psychometric properties on community and clinical samples. J Anxiety Disord 23:204-211

68. Garner DM (2004) Eating disorder inventory-3 professional manual. Psychological Assessment Resources Inc., Lutz, FL

69. Giannini M, Pannocchia P, Dalle Grave R, Muratori F, Vignone V (2008) Eating Disorder Inventory-3: Italian version. Edizioni Giunti O.S, Firenze

70. Waldherr K, Favaro A, Santonastaso P, van Strien T, Rathner G (2008) Comparison of the eating disorder inventory (EDI) in the Netherlands, Austria and Italy. Eur Eat Disord Rev 16:472-479

71. Novara C, Pardini S, Pastore M, Mulatti C (2017) Ortoressia nervosa: Un'indagine del costrutto e delle caratteristiche psicometriche della versione italiana dell'Eating Habits Questionnaire21(EHQ-21) [Orthorexia nervosa: an investigation of the construct and psychometric properties of the Italian version of the eating habits questionnaire-21 (EHQ-21).]. Psicoter Cogn E Comportamentale 23:291-316

72. R Core Team (2019) R: A language and environment for statistical computing. R Foundation for Statistical Computing, Vienna, Austria

73. Alboukadel Kassambara (2018) ggpubr: "ggplot2" based publication ready plots. R package version 0.2 . https://CRAN.R-project. org/package $=$ ggpubr

74. Kamil Barton (2019) MuMIn: multi-model inference. R package version 1.43.6. https://CRAN.R-project.org/package $=$ MuMIn

75. Kruschke J (2014) Doing Bayesian data analysis: a tutorial with R, JAGS, and Stan. Academic Press
76. Raftery AE (1995) Bayesian model selection in social research. Sociol Methodol 25:111

77. Angold A, Erkanli A, Silberg J, Eaves L, Costello EJ (2002) Depression scale scores in 8-17-year-olds: effects of age and gender. J Child Psychol Psychiatry 43:1052-1063

78. Piovesan C, Antunes JLF, Guedes RS et al (2010) Impact of socioeconomic and clinical factors on child oral health-related quality of life (COHRQoL). Qual Life Res 19:1359-1366

79. Potterton R, Richards K, Allen K, Schmidt U (2020) Eating disorders during emerging adulthood: a systematic scoping review. Front Psychol 10:3062

80. Gramaglia C, Gambaro E, Delicato C, Marchetti M, Sarchiapone M, Ferrante D et al (2019) Orthorexia nervosa, eating patterns and personality traits: a cross-cultural comparison of Italian, Polish and Spanish university students. BMC Psychiatry 19:235

81. White M, Berry R, Rodgers RF (2020) Body image and body change behaviors associated with orthorexia symptoms in males. Body Image 34:46-50

82. Rudolph S (2018) The connection between exercise addiction and orthorexia nervosa in German fitness sports. Eat Weight Disord Stud Anorex Bulim Obes 23:581-586

83. Hayes O, Wu MS, De Nadai AS, Storch EA (2017) Orthorexia nervosa: an examination of the prevalence, correlates, and associated impairment in a university sample. J Cogn Psychother 31:124-135

84. Yakın E, Raynal P, Chabrol H (2021) Distinguishing orthorexic behaviors from eating disordered and obsessive-compulsive behaviors: a typological study. Eat Weight Disord - Stud Anorex Bulim Obes 26:2011-2019

85. Novara C, Pardini S, Maggio E, Mattioli S, Piasentin S (2021) Orthorexia Nervosa: over concern or obsession about healthy food? Eat Weight Disord - Stud Anorex Bulim Obes. https://doi. org/10.1007/s40519-021-01110-x

Publisher's Note Springer Nature remains neutral with regard to jurisdictional claims in published maps and institutional affiliations. 